\title{
Elastic electron scattering using the finite element method
}

\section{Forward and inverse problems}

\author{
Denis Aubry — Ann-Lenaig Hamon — Guillaume Puel \\ Laboratoire MSSMat (Ecole Centrale Paris - CNRS UMR 8579) \\ Grande Voie des Vignes, F-92290 Châtenay-Malabry \\ \{denis.aubry, ann-lenaig.hamon, guillaume.puel\}@ecp.fr
}

\begin{abstract}
We address here the case of electron-matter elastic interaction as it occurs in Transmission Electron Microscopy (TEM) experiments. In the forward problem, we show that it is possible to derive the scattered electron wave function as the solution of a Helmholtz equation. This equation depends on the spatial potential associated with the analyzed sample, and can be relevantly solved using the Finite Element Method (FEM). Then we present an inverse formulation dealing with the determination of the sample's potential when the total wave function is measured at the exit plane of the sample.

RÉSUMÉ. Nous nous intéressons ici au cas de l'interaction élastique électron-matière rencontrée dans un microscope électronique en transmission (MET). À partir du potentiel spatial caractérisant l'échantillon observé, nous montrons que le problème direct permettant d'obtenir la fonction d'onde électronique à la sortie de l'échantillon peut s'écrire comme une équation de Helmholtz, qui peut être résolue de façon pertinente par la méthode des éléments finis (MEF). Une formulation du problème inverse qui a pour but de retrouver le potentiel de l'échantillon à partir de la fonction d'onde mesurée à la sortie de l'échantillon est également présentée.

KEYWORDS: elastic scattering, TEM, inverse problem, adjoint state, regularization.

MOTS-CLÉS : diffusion élastique, MET, problème inverse, état adjoint, régularisation.
\end{abstract}

DOI:10.3166/EJCM.19.117-128 (C) 2010 Lavoisier, Paris 


\section{Introduction}

Problems dealing with wave scattering in a heterogeneous medium are investigated in applications, whose associated scales can be extremely different (seismic waves, ultrasound waves, etc.), and are typically solved using the Finite Element Method (FEM), whatever the scale of the problem may be (see for example (Epanomeritakis et al., 2008) for the case of seismic waves). Here we want to address the case of electron-matter elastic interaction as it occurs in Transmission Electron Microscopy (TEM) experiments (Smith et al., 1982). The associated goal is to determine the electronic structure of a sample by studying how it scatters an electron wave.

In the forward problem, we assume that we know the spatial potential field associated with the sample on the electronic scale, and we calculate on the atomic scale the scattering of the electron wave function. By introducing non-restrictive simplifying assumptions, it is possible, even on this scale, to use the FEM to carry out the calculation with a reasonable computational cost. Then the obtained numerical result can be compared with scattering information measured in TEM.

To go further, we can define the following inverse problem to determine the electronic structure of the studied sample: we look for the spatial potential field that leads to a numerically calculated scattered electron wave function, which is as close as possible to the experimental one. The solutions of an inverse problem, however, are well known to be unstable and not unique. As we have shown in (Puel et al., 2008), the determination of a spatially-variable field of properties consists in an inverse problem that is awkward to regularize, particularly with typical Tikhonov regularization terms. Therefore we propose here a strategy inspired from (Bangerth et al., 2007) and based on a specific spatial discretization of the sample's potential field. This field is numerically sought by means of a mesh that is independent from the mesh used for the resolution of the wave scattering problem. This specific mesh is initially coarse, in order to regularize the inverse problem, but can then be iteratively refined by using local error estimators classically used in mesh adaption, to increase the accuracy of the identified spatial potential field.

\section{Theoretical framework of the forward electron scattering problem}

Classically, the incident electron can be considered as a complex plane wave function associated with a given real wave vector $\mathbf{k}_{i}$ :

$$
\psi_{i}(\mathbf{x})=\tilde{\psi}_{i} \exp \left(\mathrm{ik}_{i} \cdot \mathbf{x}\right)
$$

when the time harmonic factor is removed. In an empty domain $\Omega_{e}$, this wave function satisfies the following Schrödinger equation, expressed in atomic units (a.u., distances in Bohr and energies in Hartree):

$$
-\frac{1}{2} \Delta_{\mathbf{x}} \psi_{i}=E_{i} \psi_{i}
$$


where $E_{i}=\left\|\mathbf{k}_{i}\right\|^{2} / 2$ is the energy of the incident electron, and $\Delta_{\mathbf{x}}$ is the Laplacian operator with respect to the space variable $\mathbf{x}$. The domain $\Omega_{s}$ corresponding to the sample is characterized by a spatial potential field $V_{s}$, associated with the sample's electrons and nuclei, which vanishes rapidly outside $\Omega_{s}$.

The complete problem to solve should consider the incident electron as well as the sample's particules in the following generic Schrödinger equation:

$$
-\frac{1}{2}\left(\Delta_{\mathbf{x}}+\Delta_{\mathbf{x}_{s}}\right) \psi_{e s}+\left[V_{e s}\left(\mathbf{x}, \mathbf{x}_{s}\right)+V_{s}\left(\mathbf{x}_{s}\right)\right] \psi_{e s}=E \psi_{e s}
$$

where $\psi_{e s}=\psi_{e s}\left(\mathbf{x}, \mathbf{x}_{s}\right)$ is the total wave function associated with the total energy $E$ of the system. The sample's potential is expressed as the sum of two potentials $V_{s}$ and $V_{e s}$ corresponding to the sample's self-interaction and the incident electron's interaction with the sample respectively. $\mathbf{x}$ and $\mathbf{x}_{s}$ stand for the space variable associated with the incident electron and all the other particles respectively.

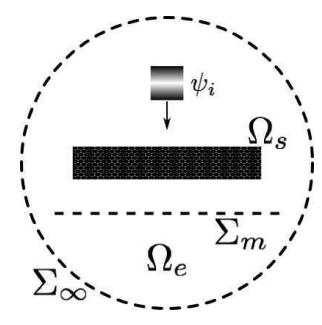

Figure 1. Considered domain for the electron scattering problem

In order to decouple the terms related to the incident electron from those associated with the sample, we use the result from (Wang, 1995) consisting in approximating the total wave function $\psi_{e s}$ as the product of two wave functions $\psi_{s}$ and $\psi_{e}$ associated with the sample and the incident electron respectively. As a result, $\psi_{e}$ should satisfy the following equation:

$$
-\frac{1}{2} \Delta_{\mathbf{x}} \psi_{e}(\mathbf{x})+V(\mathbf{x}) \psi_{e}(\mathbf{x})=\left(E-E_{s}\right) \psi_{e}(\mathbf{x})
$$

where $E_{s}$ is the sample's energy and $V$ is the sample's potential as seen by the incident electron:

$$
V(\mathbf{x})=\int_{\Omega_{s}} V_{e s}\left(\mathbf{x}, \mathbf{x}_{s}\right)\left\|\psi_{s}\left(\mathbf{x}_{s}\right)\right\|^{2} \mathrm{~d} \mathbf{x}_{s}
$$

In the particular situation of inelastic scattering, when $\left\|\mathbf{k}_{i}\right\|$ is not constant across the domain, the latter system has to be solved as a whole, and quantum transitions occurring inside the sample can be theoretically observed (Schattschneider et al., 2009). On the contrary, in the case of elastic scattering that we want to address here, it is then 
possible to simplify the previous equation using an approximation commonly used in TEM: the incident electron's velocity is very large, so that the energy $E-E_{s}$ can be approximated by the incident electron's energy $E_{i}$, which is known. In addition, it is possible to go further by expressing the electron wave function as the sum of the known incident wave $\psi_{i}$ and of the unknown scattered wave $\psi_{d}$. The scattered electron wave function $\psi_{d}$ has to satisfy the following Helmholtz equation:

$$
\frac{1}{2} \Delta \psi_{d}+E_{i} \psi_{d}=V \psi_{i}
$$

where we have considered that $V$ is small when compared to $E_{i}$.

In the conventional TEM environment, the outgoing wave $\psi_{i}+\psi_{d}$ is magnified by a series of magnetic lenses allowing an analysis both in imaging or in diffraction mode (i.e. with a Fourier transform of the wave). The intensity of the final wave $\psi_{f}$ is then collected on devices such as a screen, an imaging plate or a CCD camera. In this case, $\psi_{f}$ results from the convolution of $\psi_{i}+\psi_{d}$ with the TEM's transfer function, which mostly takes into account the effects of the defocus and of the objective lens spherical aberration. In the following, we will not address these defects, and, disregarding given scale and rotation factors, we will consider as the outgoing wave intensity the electron wave function's square module $\left\|\psi_{e}\right\|^{2}=\left\|\psi_{i}+\psi_{d}\right\|^{2}$ calculated on a virtual plane $\Sigma_{m}$ located at the exit side of the sample, as depicted in Figure 1.

\section{Numerical resolution of the forward electron scattering problem}

From the previous section, it is obtained that the forward problem consists in solving the Helmholtz Equation [6] for the scattered electron wave function $\psi_{d}$.

\subsection{The classical approach vs. the FE computation}

The classical approach consists in considering the Green's function associated with the Helmholtz equation to be solved:

$$
g(\mathbf{r})=\frac{\exp (\mathrm{ik} \cdot \mathbf{r})}{\|\mathbf{r}\|}
$$

After multiplying Equation [6] with $g$ and integrating by parts, the scattered wave function $\psi_{d}$ can be expressed as:

$$
\psi_{d}(\mathbf{x})=\int_{\mathbb{R}^{3}} V(\mathbf{y}) \psi_{i}(\mathbf{y}) g(\mathbf{x}-\mathbf{y}) \mathrm{d} \mathbf{y}=\left(\left(V \psi_{i}\right) * g\right)(\mathbf{x})
$$

In practice, $\psi_{d}$ is then calculated using the multislice method, which divides the samples into several slices with respect to the thickness (Ishizuka et al., 1977; Stadelmann, 1987; Williams et al., 1996; Kirkland, 1998). Although classically used, this method necessitates a more or less empirical choice of these slices' size, and is not 
able to take into account the waves that may be reflected back and forth between the atoms.

Instead of using the previous method, we prefer to consider an approach that is able to solve Equation [6] without further assumptions. This approach should then be able to deal with non periodical samples (i.e. with a single defect) as well as to take into account all the waves interacting in the problem.

Therefore we propose to use the FEM, although this requires some adaptations. The first one is related to the fact that the initial problem [6] is defined on a domain $\Omega_{e}$ whose dimensions are infinite. In spite of this, it can be shown that the scattered wave function $\psi_{d}$ is of the evanescent kind far from the considered sample, which allows to bound the domain $\Omega_{e}$ with a boundary denoted $\Sigma_{\infty}$ in Figure 1. On this boundary the following evanescent condition is applied, as proposed in (Popov, 2006):

$$
\frac{\partial \psi_{d}}{\partial \mathbf{n}}=\mathrm{i}\left\|\mathbf{k}_{i}\right\| \psi_{d}
$$

where $\mathbf{n}$ stands for the unit outward normal along the boundary $\Sigma_{\infty}$.

The main difficulty, however, lies in the high-frequency content of the problem to be solved. While interatomic distances are about 5 a.u. (and sample's thickness about $10^{5}$ a.u.), an estimate of the incident wave length is $\lambda_{i}=0.05$ a.u. If we assume that $10 \mathrm{FE}$ degrees of freedom par wave length are to be used in order to accurately discretize the calculated wave, an amount of $10^{9}$ degrees of freedom is required to mesh a single crystalline cell, which would be impractical as such without additional adaptations.

\subsection{Paraxial approximation}

In order to deal with the high-frequency content of $\psi_{d}$, the paraxial approximation consists in searching for the unknown $\tilde{\psi}_{d}$ such that:

$$
\psi_{d}(\mathbf{x})=\tilde{\psi}_{d}(\mathbf{x}) \exp \left(\mathrm{ik}_{i} \cdot \mathbf{x}\right)
$$

where $\mathbf{k}_{i}$ is the incident wave vector, along the microscope's axis in the case of parallel illumination (which corresponds to classical experimental conditions). Even if, formally, this approximation does not imply that the scattered wave function should be oriented along the incident wave's direction, this fits well with the TEM's experimental conditions, where all the rays that are diffracted with an angle greater than about $30 \mathrm{mrad}$ are truncated by the microscope's transfer function.

By using the approximation [10] into Equation [6], one gets:

$$
\frac{1}{2} \Delta \tilde{\psi}_{d}+\mathbf{i k}_{i} \cdot \nabla \tilde{\psi}_{d}=V \tilde{\psi}_{i} \text { in } \Omega_{e}
$$

because $E_{i}=\left\|\mathbf{k}_{i}\right\|^{2} / 2$. Similarly, the evanescent condition [9] gives, with [10]:

$$
\frac{\partial \tilde{\psi}_{d}}{\partial \mathbf{n}}=\mathrm{i}\left(\left\|\mathbf{k}_{i}\right\|-\mathbf{k}_{i} \cdot \mathbf{n}\right) \tilde{\psi}_{d} \text { on } \Sigma_{\infty}
$$




\subsection{Numerical FE resolution}

The numerical resolution of the previous group of equations first consists in choosing finite dimensional spaces $\mathcal{V}_{h}$ and $\mathcal{W}_{h}$ using typical FE discretizations associated with a given mesh $\mathcal{M}_{h}$. Then the discrete forward problem consists in finding $\tilde{\psi}_{d, h} \in \mathcal{W}_{h}$ such that:

$$
\begin{array}{r}
\int_{\Omega_{e}}\left(-\frac{1}{2} \nabla \tilde{\psi}_{d, h} \cdot \nabla w_{h}^{*}+\mathrm{i} \mathbf{k}_{i} \cdot \nabla \tilde{\psi}_{d, h} w_{h}^{*}\right) \\
+\int_{\Sigma_{\infty}} \frac{\mathrm{i}}{2}\left(\left\|\mathbf{k}_{i}\right\|-\mathbf{k}_{i} \cdot \mathbf{n}\right) \tilde{\psi}_{d, h} w_{h}^{*}=\int_{\Omega_{e}} V_{h} \tilde{\psi}_{i, h} w_{h}^{*} \quad \forall w_{h} \in \mathcal{W}_{h}
\end{array}
$$

where $\nabla$ and $\cdot^{*}$ stand for the gradient operator and the complex conjugate respectively. $V_{h} \in \mathcal{V}_{h}$ is the spatial discretization of the sample's potential $V$ on the mesh $\mathcal{M}_{h}$.

As a example to demonstrate the validity of the proposed approach, we choose a very thin 2D sample made of pure $\alpha$-iron. The potential $V$ associated with the crystal is simulated by means of a Yukawa's potential (Ashkroft et al., 2002):

$$
V=\sum_{k=1}^{N} V_{a} \frac{\exp \left(-a r_{k}\right)}{r_{k}}
$$

where $r_{k}$ stands for the distance from the $k$-th nucleus (out of $N$ nuclei), and $V_{a}$ and $a$ are two constants to set. This potential is discretized on the mesh $\mathcal{M}_{h}$.

(a)

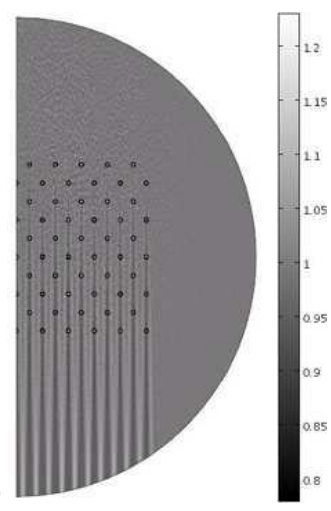

(b)

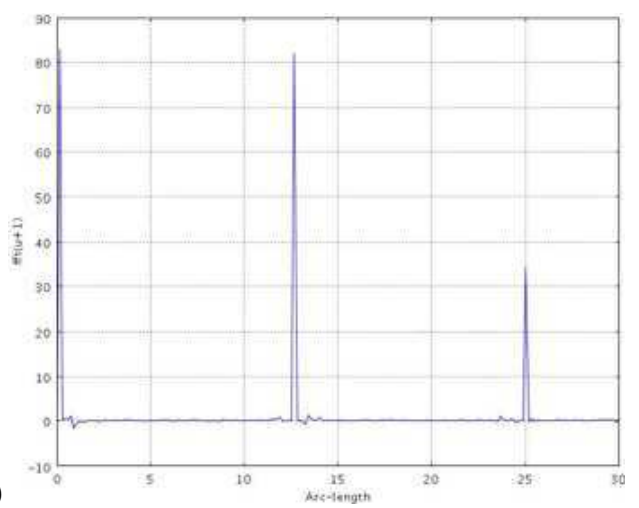

Figure 2. (a) FE forward calculation of $\left\|\psi_{e}\right\|^{2}=\left\|\psi_{i}+\psi_{d}\right\|^{2}$ for an $\alpha$-iron sample (b) FFT of the outgoing wave function $\psi_{i}+\psi_{d}$ on the virtual plane $\Sigma_{\infty}$

Figure 2 shows the calculation with 600,000 degrees of freedom of the intensity $\left\|\psi_{e}\right\|^{2}=\left\|\psi_{i}+\psi_{d}\right\|^{2}$ in a $200 \mathrm{keV}$-microscope with $\left\|\mathbf{k}_{i}\right\|=130 \mathrm{rad} /($ a.u. $)$. The chosen sample, whose thickness $(2 \mathrm{~nm})$ is below actual experimental values, is oriented along the [001] direction, and the associated Yukawa's potential is represented 
in Figure 2 with isolines representing the location of the sample's nuclei. Since Equation [13] is linear with respect to the incident wave's amplitude $\tilde{\psi}_{i, h}$ as well as the potential's amplitude $V_{a}$, both values are set equal to 1 . In addition, an absorption coefficient of $5.10^{-3}$ is introduced to improve the convergence of the calculation. Eventually the Fast Fourier Transform (FFT) of the outgoing wave function $\psi_{i}+\psi_{d}$ is depicted in Figure 2. Diffracted beams clearly appear, according to Bragg's law relative to the interatomic distances and the incident wave length $\lambda_{i}$.

\section{Formulation of the inverse electron scattering problem}

The inverse problem consists in determining in $\Omega_{e}$ the spatial potential field $V$ such that the electronic wave's intensity $\left\|\tilde{\psi}_{i}+\tilde{\psi}_{d}\right\|^{2}$, which is numerically calculated with $V_{h}$ on the virtual plane $\Sigma_{m}$, best fits the measured intensity $\left\|\psi_{m}\right\|^{2}$ of the outgoing wave. By this means, it should be possible to detect a defect within the studied sample.

The usual technique is to introduce the discrepancy between the calculated and the measured intensities through a misfit function (Beilina et al., 2005; Beilina et al., 2006):

$$
\mathcal{J}(V)=\frac{1}{4} \int_{\Sigma_{m}}\left(\left\|\tilde{\psi}_{i}+\tilde{\psi}_{d}\right\|^{2}-\left\|\psi_{m}\right\|^{2}\right)^{2}+\frac{\alpha}{2} \int_{\Omega_{v}}\left(V-V_{0}\right)^{2}
$$

where $\alpha$ is a regularization parameter to be set, and $\Omega_{v} \subset \Omega_{e}$ the domain where the potential $V$ is looked for. $V_{0}$ is a potential field, which is chosen a priori. Typically this latter is assumed to be close to the sought potential field: for example, when dealing with the detection of a defect within the sample, one can choose the potential associated with the perfect crystal. In this latter case, the inverse problem consists in finding the defect by means of the involved modification of the potential.

\subsection{Adjoint state formulation}

The minimization of the previous misfit function [15] is usually performed by means of gradient-based techniques. In oder to avoid time-consuming calculations as well as inaccuracies associated with numerical differentiation, the derivative of $\mathcal{J}(V)$ is analytically introduced by means of an adjoint state problem. The solution $z$ of this adjoint state problem can be considered as a Lagrange multiplier introduced in the following Lagrangian function $\mathcal{L}\left(\tilde{\psi}_{d}, V, z\right)$ :

$$
\begin{array}{r}
\mathcal{L}\left(\tilde{\psi}_{d}, V, z\right)=\frac{1}{4} \int_{\Sigma_{m}}\left(\left\|\tilde{\psi}_{i}+\tilde{\psi}_{d}\right\|^{2}-\left\|\psi_{m}\right\|^{2}\right)^{2}+\frac{\alpha}{2} \int_{\Omega_{v}}\left(V-V_{0}\right)^{2} \\
+\operatorname{Re}\left\{\int_{\Omega_{e}}\left(-\frac{1}{2} \nabla \tilde{\psi}_{d} \cdot \nabla z^{*}+\left(\mathbf{i} \mathbf{k}_{i} \cdot \nabla \tilde{\psi}_{d}-V \tilde{\psi}_{i}\right) z^{*}\right)\right\} \\
+\operatorname{Re}\left\{\int_{\Sigma_{\infty}} \frac{\mathrm{i}}{2}\left(\left\|\mathbf{k}_{i}\right\|-\mathbf{k}_{i} \cdot \mathbf{n}\right) \tilde{\psi}_{d} z^{*}\right\}
\end{array}
$$


where $\left(\tilde{\psi}_{d}, V, z\right)$ are considered as independent and Re stands for the real part. Minimizing $\mathcal{J}(V)$ with $\tilde{\psi}_{d}$ verifying Equations [11]-[12] is then equivalent to writing the first-order stationarity conditions for $\mathcal{L}\left(\tilde{\psi}_{d}, V, z\right)$.

The first-order stationarity condition with respect to $\tilde{\psi}_{d}$ leads to the adjoint state problem, which is very close to the forward problem:

$$
\begin{aligned}
& \frac{1}{2} \Delta z+\mathrm{i} \mathbf{k}_{i} \cdot \nabla z=0 \text { in } \Omega_{e} \\
& \frac{\partial z}{\partial \mathbf{n}}=-\mathrm{i}\left(\left\|\mathbf{k}_{i}\right\|+\mathbf{k}_{i} \cdot \mathbf{n}\right) z \text { on } \Sigma_{\infty} \\
& \frac{1}{2}\left[\left[\frac{\partial z}{\partial \mathbf{n}}\right]\right]=\left(\left\|\tilde{\psi}_{i}+\tilde{\psi}_{d}\right\|^{2}-\left\|\psi_{m}\right\|^{2}\right)\left(\tilde{\psi}_{i}+\tilde{\psi}_{d}\right) \text { on } \Sigma_{m}
\end{aligned}
$$

where $[[\cdot]]$ stands for the discontinuity gap. The adjoint state can be interpreted as the solution of a backwards wave scattering problem.

Then the first-order derivative of $\mathcal{L}\left(\tilde{\psi}_{d}, V, z\right)$ with respect to $V$ allows us to express the directional derivative of the misfit function easily:

$$
\begin{aligned}
D_{V} \mathcal{J}(V) \delta V & =D_{V} \mathcal{L}\left(\tilde{\psi}_{d}, V, z\right) \delta V \\
& =\int_{\Omega_{v}}\left(\alpha\left(V-V_{0}\right)-\operatorname{Re}\left(\tilde{\psi}_{i}^{*} z^{*}\right)\right) \delta V
\end{aligned}
$$

\subsection{Numerical resolution of the inverse problem}

The minimum of the misfit function $\mathcal{J}(V)$ is sought as $D_{V} \mathcal{J}(V) \delta V=0 \forall \delta V$, which could be rewritten as the following compatibility equation:

$$
\operatorname{Re}\left(\tilde{\psi}_{i}^{*} z^{*}\right)=\alpha\left(V-V_{0}\right) \text { in } \Omega_{v}
$$

The regularization described in [15] does not consider at all the issue of global minimizers. Conditions to insure their existence are provided by (Chavent, 1991). The minimization problem eventually consists in solving three Partial Differential Equations with unknowns $\left(\tilde{\psi}_{d}, V, z\right)$ : the forward problem [11]-[12], the adjoint problem [17]-[18]-[19] and the compatibility Equation [21]. The identification process results in the resolution of a system, which is highly nonlinear in the spatially-variable unknown field $V$.

The FE numerical resolution then consists in finding $\left(\tilde{\psi}_{d, h}, V_{h}, z_{h}\right) \in \mathcal{W}_{h} \times \mathcal{V}_{h} \times$ $\mathcal{W}_{h}$ such that:

$$
\begin{array}{r}
\int_{\Omega_{e}}\left(-\frac{1}{2} \boldsymbol{\nabla} \tilde{\psi}_{d, h} \cdot \nabla w_{h}^{*}+\mathbf{i k}_{i} \cdot \nabla \tilde{\psi}_{d, h} w_{h}^{*}\right) \\
+\int_{\Sigma_{\infty}} \frac{\mathrm{i}}{2}\left(\left\|\mathbf{k}_{i}\right\|-\mathbf{k}_{i} \cdot \mathbf{n}\right) \tilde{\psi}_{d, h} w_{h}^{*}=\int_{\Omega_{e}} V_{h} \tilde{\psi}_{i, h} w_{h}^{*} \quad \forall w_{h} \in \mathcal{W}_{h}
\end{array}
$$




$$
\begin{array}{r}
\int_{\Omega_{e}}\left(-\frac{1}{2} \boldsymbol{\nabla} z_{h} \cdot \boldsymbol{\nabla} w_{h}^{*}-\mathrm{i} \mathbf{k}_{i} \cdot \nabla z_{h} w_{h}^{*}\right)+\int_{\Sigma_{\infty}} \frac{\mathrm{i}}{2}\left(\left\|\mathbf{k}_{i}\right\|+\mathbf{k}_{i} \cdot \mathbf{n}\right) z_{h} w_{h}^{*} \\
+\int_{\Sigma_{m}}\left(\left\|\tilde{\psi}_{i, h}+\tilde{\psi}_{d, h}\right\|^{2}-\left\|\psi_{m}\right\|^{2}\right)\left(\tilde{\psi}_{i, h}+\tilde{\psi}_{d, h}\right) w_{h}^{*}=0 \quad \forall w_{h} \in \mathcal{W}_{h} \\
\int_{\Omega_{v}}\left(\alpha\left(V_{h}-V_{0, h}\right)-\operatorname{Re}\left(\tilde{\psi}_{i, h}^{*} z_{h}^{*}\right)\right) \delta V_{h}=0 \quad \forall \delta V_{h} \in \mathcal{V}_{h}
\end{array}
$$

For the time being, instead of actual experimental data, we use synthetic data such as those obtained with Equation [13]. The resolution of the inverse problem "as is" can lead to some difficulties, mainly coming from the fact that we want to determine a spatially-variable represented by a large amount of scalar values to be identified, whereas experimental information is scarce. The mesh used for the discretization of this field may then influence the resolution of the inverse problem, and even if it is not the case, using a mesh which has to be fine enough to deal with the calculation of the forward and adjoint solutions can lead to a very costly identification process.

\subsection{Iterative strategy using two different meshes}

For all these reasons, we propose to apply the strategy inspired from (Bangerth et al., 2007) and described in (Puel et al., 2008), where the spatial discretization of the field to be identified is achieved with a mesh different from the one associated with the calculation of the forward and adjoint solutions. So we introduce two distinct meshes: a sufficiently refined mesh $\mathcal{M}_{h}$ for the resolution of the forward and adjoint problems [22]-[23], and a coarse mesh $\mathcal{M}_{H}$ for the discretization of the sought field $V$ and the resolution of the compatibility equation [24]. Then the discrete problem consists in finding $\left(\tilde{\psi}_{d, h}, V_{H}, z_{h}\right) \in \mathcal{W}_{h} \times \mathcal{V}_{H} \times \mathcal{W}_{h}$ such that:

$$
\begin{array}{r}
\int_{\Omega_{e}}\left(-\frac{1}{2} \nabla \tilde{\psi}_{d, h} \cdot \nabla w_{h}^{*}+\mathrm{i} \mathbf{k}_{i} \cdot \nabla \tilde{\psi}_{d, h} w_{h}^{*}\right) \\
+\int_{\Sigma_{\infty}} \frac{\mathrm{i}}{2}\left(\left\|\mathbf{k}_{i}\right\|-\mathbf{k}_{i} \cdot \mathbf{n}\right) \tilde{\psi}_{d, h} w_{h}^{*}=\int_{\Omega_{e}} \Pi_{h}^{H} V_{H} \tilde{\psi}_{i, h} w_{h}^{*} \quad \forall w_{h} \in \mathcal{W}_{h} \\
\int_{\Omega_{e}}\left(-\frac{1}{2} \nabla z_{h} \cdot \nabla w_{h}^{*}-\mathrm{i} \mathbf{k}_{i} \cdot \nabla z_{h} w_{h}^{*}\right)+\int_{\Sigma_{\infty}} \frac{\mathrm{i}}{2}\left(\left\|\mathbf{k}_{i}\right\|+\mathbf{k}_{i} \cdot \mathbf{n}\right) z_{h} w_{h}^{*} \\
+\int_{\Sigma_{m}}\left(\left\|\tilde{\psi}_{i, h}+\tilde{\psi}_{d, h}\right\|^{2}-\left\|\psi_{m}\right\|^{2}\right)\left(\tilde{\psi}_{i, h}+\tilde{\psi}_{d, h}\right) w_{h}^{*}=0 \quad \forall w_{h} \in \mathcal{W}_{h} \\
\int_{\Omega_{v}}\left(\alpha\left(V_{H}-V_{0, H}\right)-\operatorname{Re}\left(\tilde{\psi}_{i, H}^{*} \Pi_{H}^{h} z_{h}^{*}\right)\right) \delta V_{H}=0 \quad \forall \delta V_{H} \in \mathcal{V}_{H}
\end{array}
$$

where $\mathcal{W}_{h}$ and $\mathcal{V}_{H}$ are associated with the fine mesh $\mathcal{M}_{h}$ and the coarse mesh $\mathcal{M}_{H}$ respectively. $\Pi_{H}^{h}: \mathcal{W}_{h} \rightarrow \mathcal{W}_{H}$ and $\Pi_{h}^{H}: \mathcal{V}_{H} \rightarrow \mathcal{V}_{h}$ are specific operators (projection and extension respectively). 
Of course, the identified potential field $V_{H}$ is not very accurate for $\mathcal{M}_{H}$ is chosen coarse to regularize the inverse problem. To improve the identification further, we propose an iterative method based on Bangerth's work (Bangerth et al., 2007): the mesh $\mathcal{M}_{H}$ used for the discretization of the spatial field $V$ is progressively refined according to classical mesh adaption methods. These latter rely on a posteriori error estimators, such as estimators quantifying the quality of a mesh regarding the reference continuous mechanical problem. For implementation purposes, we choose the classical L2-norm error indicator based on the residual $r$ associated with Equation [27] as defined in (Eriksson et al., 1996) and (Verfurth, 1996):

$$
e_{L 2}=\left(\int_{\Omega} H^{4}|r|^{2} \mathrm{~d} \Omega\right)^{\frac{1}{2}}
$$

where $H$ is the local size of the mesh $\mathcal{M}_{H}$. This error indicator can be split into local contributions for every element of the mesh $\mathcal{M}_{H}$. Elements whose contribution belongs to the highest ones (e.g. 5\%) are refined. The adaption steps stop when the first term of the misfit function [15] is below a given threshold indirectly characterizing the quality of the identified spatial field $V$.

Of course, it would be possible to use similar L2-norm error indicators to refine the mesh $\mathcal{M}_{h}$ as well. Here, however, this choice is not made for implementation purposes, and we assume that the mesh $\mathcal{M}_{h}$ is sufficiently refined for the resolution of the forward and adjoint problems [25] and [26].

\section{4. $2 D$ example}

The previous strategy is applied to the detection of a defect within a given sample. First, synthetic data are obtained with Equation [13] using a sample with a lacuna as seen in Figure 3; it is simply assumed that the sample's potential corresponds to the perfect crystal's potential minus the potential associated with the missing atom.

Concerning the resolution of the inverse problem, $\alpha$ is set so that both terms in the misfit function [15] have approximately the same magnitude: $\alpha \approx \frac{\left|\Sigma_{m}\right||| \psi_{m}||_{\infty}^{4}}{2\left|\Omega_{v}\right||| V_{0}||_{\infty}^{2}}$. The mesh $\mathcal{M}_{h}$ associated with the forward and adjoint problems consists of 5,044 quadratic elements, whereas the initial mesh $\mathcal{M}_{H}^{0}$ discretizing the difference $\Delta V=V-V_{0}$ between the sought potential and the perfect crystal's potential is made of 8 linear elements, which constitute the search domain $\Omega_{v}$ enclosing the crystal. Both meshes are depicted in Figure 4.

Figure 3 shows the identified potential difference $\Delta V$ after 5 refinement steps, while the associated mesh $\mathcal{M}_{H}^{5}$ made of 1,201 linear elements is visible in Figure 4. The strongest fluctuations are located in the vicinity of the lacuna, but several artefacts are visible, mainly close to the boundaries of the search domain $\Omega_{v}$. This can be an effect of the regularization, which is all the more awkward to set in the present case where experimental data are scarce comparatively with the complexity of the spatial potential to be identified. 
(a)

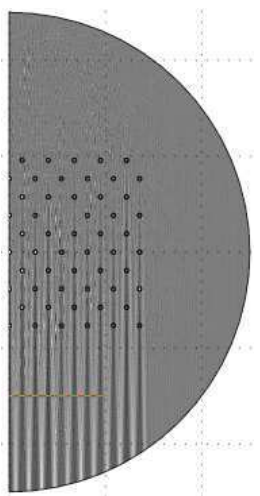

(b)

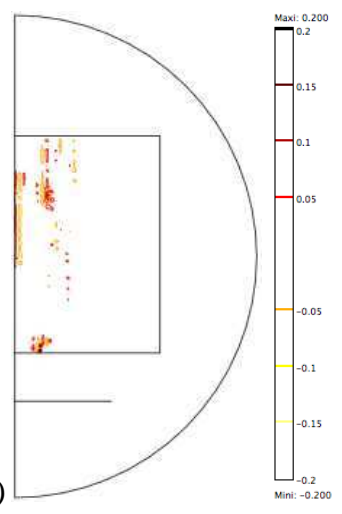

Figure 3. (a) FE forward calculation of $\left\|\psi_{e}\right\|^{2}=\left\|\psi_{i}+\psi_{d}\right\|^{2}$ for an $\alpha$-iron sample with a lacuna $(b)$ Identified potential difference $\Delta V$ after 5 iterations

(a)

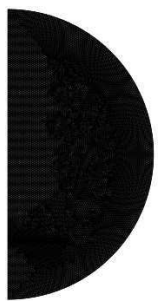

(b)

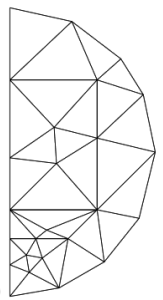

(c)

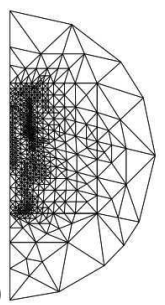

Figure 4. (a) Mesh $\mathcal{M}_{h}$ associated with $\tilde{\psi}_{d, h}\left(\right.$ b) Initial mesh $\mathcal{M}_{H}^{0}$ associated with $V_{H}-V_{0}(c)$ Mesh $\mathcal{M}_{H}^{5}$ after 5 iterations

\section{Conclusion}

First we showed that electron-matter elastic interaction as it occurs in TEM experiments can be numerically solved using the FEM. With some non-restrictive assumptions and adaptations, the forward elastic electron scattering can be reduced into a Helmholtz equation that can be efficiently solved using a paraxial approximation. We then obtain the intensity of the total interacting wave after it crossing the sample.

When dealing with the inverse problem of identifying a crystal's potential from the intensity of the total interacting wave, one is often confronted with the difficult choice of a relevant regularization. This is particularly true when the sought spatial field is discretized on a FE mesh, for its choice can influence the result of the identification.

Here we introduce a general iterative strategy using adaptive meshes. The goal is to use a specific mesh $\mathcal{M}_{H}$ for the spatial discretization of the potential to be identified. 
Using a coarse mesh makes the regularization easier, and the identification can be improved by refining $\mathcal{M}_{H}$ according to classical error estimators. Further studies will focus on the identification strategy. In particular, the influence of the sample's size and the use of different illumination directions should be considered, as well as the choice of different regularization terms and refinement criteria.

\section{References}

Ashkroft N. W., Mermin N. D., Physique des Solides, EDP Sicences, Les Ulis, 2002.

Bangerth W., Joshi A., Sevick-Muraca E. M., “ Adaptive FE methods for increased resolution in fluorescence optical tomography", Progress in Biomedical Optics and Imaging, vol. 6, p. 318-329, 2007.

Beilina L., Clason C., “ An adaptive hybrid FEM/FDM method for an inverse scattering problem in scanning acoustic microscopy”, SIAM Journal on Scientific Computing, vol. 28, n 1 , p. 382-402, 2006.

Beilina L., Johnson C., “ A posteriori error estimation in computational inverse scattering”, Mathematical Models and Methods in Applied Sciences, vol. 15, $\mathrm{n}^{\circ}$ 1, p. 23-37, 2005.

Chavent G., " Quasi-convex sets and size $\times$ curvature condition, application to nonlinear inversion”, Applied Mathematics and Optimization, vol. 24, n 1, p. 129-169, 1991.

Epanomeritakis I., Akçelik V., Ghattas O., Bielak J., “ A Newton-CG method for large-scale three-dimensional elastic full-waveform seismic inversion”, Inverse Problems, vol. 24, n 3 , p. $034015,2008$.

Eriksson K., Estep D., Hansbo P., Johnson C., Computational Differential Equations, 2nd edn, Cambridge University Press, Cambridge, 1996.

Ishizuka K., Uyeda N., " A new theoretical and practical approach to the multislice method", Acta Crystallographica Section A, vol. 33, p. 740-749, 1977.

Kirkland E. J., Advanced Computing in Electron Microscopy, Plenum, New York, 1998.

Popov A. V., " Computation of paraxial wave fields using transparent boundary conditions", Computational Mathematics and Mathematical Physics, vol. 46, n 9, p. 1595-1600, 2006.

Puel G., Aubry D., “ Identification of a spatial field of material properties with adaptive regularization and meshes", Proceedings of IDETC/CIE 2008, ASME, New York, p. (CD-ROM), August 3-6, 2008.

Schattschneider P., Verbeeck J., Hamon A. L., “Real space maps of atomic transitions”, Ultramicroscopy, vol. 109, n 7, p. 781-787, 2009.

Smith A. R., Eyring L., " Calculation, display and comparison of electron microscope images modeled and observed", Ultramicroscopy, vol. 8, p. 65-78, 1982.

Stadelmann P. A., " EMS - a software package for electron diffraction analysis and HREM image simulation in materials science", Ultramicroscopy, vol. 21, p. 131-146, 1987.

Verfurth R., A review of a posteriori error estimation and adaptive mesh-refinement techniques, Wiley, Hoboken, 1996.

Wang Z. L., Elastic and inelastic scattering in electron diffraction and imaging, Plenum, New York, 1995.

Williams D. B., Carter C. B., Transmission electron microscopy, Plenum, New York, 1996. 